\title{
Recent Advances in EUV Solar Astronomy
}

\section{G. A. DOSCHEK}

\author{
Code 7670, E.O. Hulburt Center for Space Research, \\ Naval Research Laboratory, Washington, DC 20375-5320, USA
}

I discuss recent advances in EUV solar astronomy. The new work is primarily a result of current solar space missions such as the Yohkoh high energy solar physics mission, as well as upcoming space missions such as the ESA Solar and Heliospheric Observatory (SOHO). I discuss spectroscopic and atomic physics work, and new results concerning solar flares that are directly relevant to stellar research.

\section{Introduction}

The Sun is the only astrophysical object for which the electrodynamics and plasma physics of high temperature plasmas can be studied in detail. Amazingly, plasmas that reach equivalent kinetic temperatures of more than 100 million degrees can occur by natural processes in the solar atmosphere. Accelerated particles in these plasmas are sufficiently energetic to produce nuclear reactions and consequent gamma ray continuum and line emission in a relatively low density medium. These energetic phenomena are believed to be the result of the conversion of magnetic energy into kinetic energy, but the precise physical processes are not known. The physics of solar activity is intrinsically fascinating, even if the rest of the Universe did not exist.

There are many examples in non-solar astronomy that involve complex electrodynamical processes, e.g., astrophysical jets, magnetic fields in the interstellar medium, stellar atmospheres and violent processes in these atmospheres, compact objects with accretion disks, etc. In most of these examples the sources cannot be spatially resolved, and therefore electrodynamical theories involving these source must by necessity rely on considerable conjecture. Understanding electrodynamical processes in the solar atmosphere can replace at least some of the conjectures with documented physical insight based on physical understanding achieved through solar physics.

In a less sophisticated sense, the Sun serves as a source for high resolution emission line spectroscopy of cosmic plasmas. Because solar activity produces plasmas that span a very wide temperature range, from a few thousand to millions of degrees, spectral lines characteristic of all these temperature regions can be recorded by high resolution solar spectroscopic experiments, and in particular, very high resolution can be achieved because the line intensities are obviously much higher than in other cosmic plasmas. High resolution solar spectroscopy experiments have been carried out for about 30 years, and have been a great stimulus for the study of the spectroscopy and atomic physics of highly ionized ions. Because of solar spectroscopy, UV, EUV, and X-ray emission line spectra of cosmically abundant elements are now well-understood in terms of spectral classification and plasma diagnostic potential for deriving important physical parameters such as electron temperature and density. For example, most of the atomic physics and plasma diagnostics necessary for the analysis of emission line spectra from the current $E U V E$ and $A S C A$ missions already exists, and is continually being improved upon because of new solar space experiments, either existing or planned. Laboratory research involving tokamak plasmas has also helped with EUV and X-ray atomic physics and spectroscopy, but the main driver for the cosmically abundant elements has been, and will continue to be, the Sun. 
In this paper I highlight some recent results in EUV and X-ray spectroscopy, and discuss some surprising new flare observations that indicate the occurrence of some counterintuitive processes in flaring magnetic flux tubes.

\section{Current and Future Solar Spectroscopic Space Missions}

There is no current solar EUV spectroscopic orbiting spacecraft experiment. However, there have been several recent flights of a high spectral and spatial resolution rocket experiment, designed and built by the solar group at Goddard Space Flight Center. This instrument, the Solar EUV Rocket Telescope and Spectrograph (SERTS), has obtained calibrated high resolution spectra of solar quiet and active regions, and an extensive active region line list with intensities has been published by Thomas \& Neupert (1994). The last previous well-calibrated line list for EUV wavelengths greater than about $100 \AA$ was published by Malinovsky \& Heroux (1973), based on a rocket experiment. For EUV wavelengths less than $100 \AA$, the most recent line list with calibrated intensities is the Acton et al. (1985) list, again based on a rocket spectrograph experiment.

Major new high resolution EUV and UV results can be expected from the ESA cornerstone mission, the Solar and Heliospheric Observatory $(S O H O)$ spacecraft, to be launched around the end of 1995 . The $S O H O$ spacecraft will be in orbit around the L1 Lagrangian point and will have an unobstructed view of the Sun. The payload consists of 12 instruments furnished by different consortia. Although $S O H O$ is an ESA mission, NASA is supplying three solar experiments and the spacecraft operations and science analysis centers will be at GSFC.

SOHO contains two major experiments devoted to EUV and UV spectroscopy. The lead institution for the EUV instrument, the Coronal Diagnostics Spectrometer (CDS), is the Rutherford Appleton Laboratory. The spectrometer contains both normal and grazing incidence optics, and covers selected wavelength regions between 155 and $787 \AA$. The spectral resolving power is wavelength dependent but is very high, ranging between 3,500 and 12,000 . The spatial resolution along the slit is also quite high, about 2 arcseconds over a 4 arcminute field of view. The lead institution for the UV spectrometer, called Solar Ultraviolet Measurement of Emitted Radiation (SUMER), is the Max Planck Institute for Aeronomy. This instrument will cover the wavelength region from 500 to $1600 \AA$, with a spectral resolving power $>18,000$ and a spatial resolution along the slit of about 1 arcsecond.

Primarily because of the reflectance properties of mirrors, the simultaneous measurement of both UV and EUV lines with high resolution has not been done before in solar astronomy, and therefore many promising new plasma diagnostics can be used for the first time in the analysis of solar spectra obtained by CDS and SUMER. For example, intersystem lines of many transition region ions such as O IV and $\mathrm{O} V$ fall in the UV, but their resonance lines fall in the EUV. Combining the UV and EUV lines of these ions provides both temperature and density sensitive line ratios.

The $S O H O$ spectroscopy experiments have formed the basis for a concentrated effort by solar atomic physicists to make a quantum leap improvement in EUV and UV atomic data, and the application of these data to plasma diagnostics. The goal is to make analysis of CDS and SUMER data straightforward for solar researchers who are not trained in spectroscopy. This requires a systematic approach to atomic physics calculations and their adaptation to plasma diagnostics. These atomic physics efforts are discussed in detail elsewhere in this Proceedings.

Although there are no current EUV orbiting spacecraft solar missions, the Japanese Yohkoh high energy solar physics mission contains four X-ray Bragg crystal spectrome- 


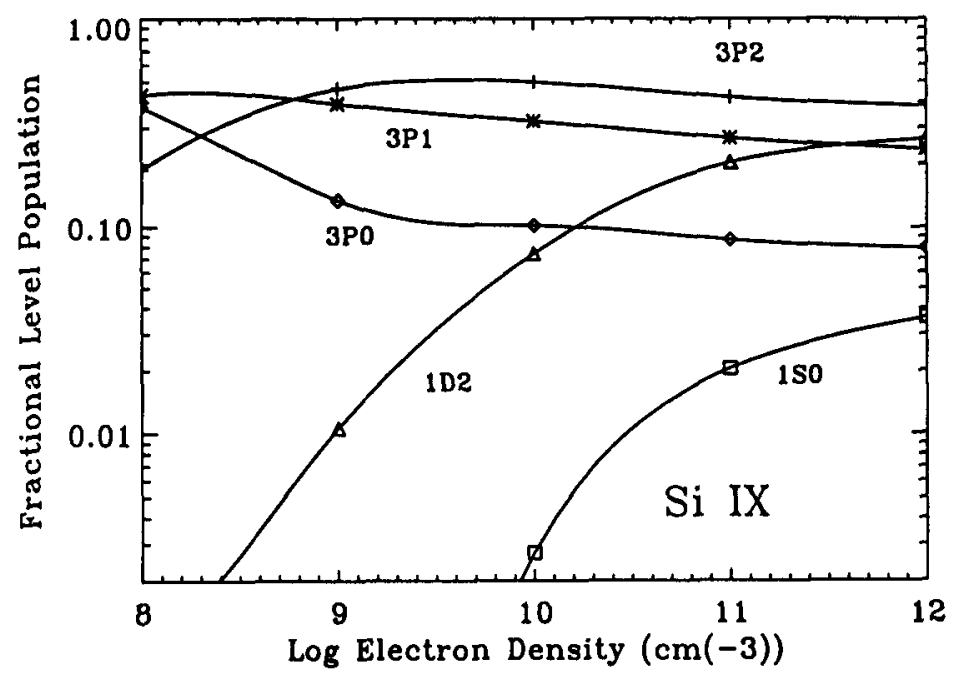

Figure 1. Ground configuration fractional level populations

ters. These spectrometers, in combination with the soft and hard X-ray telescopes on Yohkoh, have revealed some surprising properties of solar flares. Although X-ray observations, the results concern the physics of flares which transcends wavelength ranges. Some of the results are discussed in Section 4.

\section{Atomic Physics and Plasma Diagnostics}

The new atomic physics calculations carried out in support of future space missions in astronomy can be compared with the recent SERTS results (Thomas \& Neupert 1994) and the earlier results from Malinovsky \& Heroux (1973). As an illustration, consider $\mathrm{Si}$ IX of the C I isoelectronic sequence. The ground configuration, $2 \mathrm{~s}^{2} 2 \mathrm{p}^{2}$, consists of five levels, ${ }^{3} \mathrm{P}_{0,1,2},{ }^{1} \mathrm{D}_{2}$, and ${ }^{1} \mathrm{~S}_{0}$. The populations of these levels vary with electron density, which leads to density sensitivity of some of the lines at typical stellar atmosphere densities.

The theoretical level populations of the ground configuration are shown in Figure 1, and theoretical relative line intensities are given in Table 1, compared with the SERTS and Malinovsky \& Heroux (1973) data. The theoretical intensities are from Bhatia \& Doschek (1993), assuming a temperature of about $1.3 \times 10^{6} \mathrm{~K}$ for the formation of $\mathrm{Si}$ IX. The best density sensitive line is the line at $258.095 \AA$, which terminates on the ${ }^{1} \mathrm{D}_{2}$ level. In general, the theoretical relative line intensities (arbitrarily with respect to the line at $341.974 \AA$ ) agree reasonably well with the data. The density sensitive ratio indicates a density of about $10^{10} \mathrm{~cm}^{-3}$ for the SERTS active region, which seems reasonable. Although there is general agreement between theory and experiment, there are some problems, such as the $296.137 \AA$ line ratio, for which SERTS data and theory do not agree very well.

In most calculations, the relative line intensities generally agree, but sometimes there are disturbing exceptions. These exceptions may result from unresolved line blends, 
TABLE 1. Si IX Line Intensity Ratios

\begin{tabular}{llllllll} 
Wave & & \multicolumn{7}{c}{ Intensity Ratio (ergs) } \\
& SERTS & $\mathrm{MH}^{\dagger}$ & $10^{8}$ & $10^{9}$ & $10^{10}$ & $10^{11}$ & $10^{12}$ \\
$55.3^{\ddagger}$ & $\ldots$ & 3.24 & 1.60 & 3.41 & 4.03 & 4.06 & 4.08 \\
223.75 & $\ldots$ & 1.34 & 0.294 & 0.633 & 0.743 & 0.735 & 0.727 \\
$225.033^{\ddagger}$ & $\ldots$ & 2.51 & 0.874 & 1.89 & 2.22 & 2.19 & 2.16 \\
227.01 & $\ldots$ & 4.41 & 1.50 & 3.30 & 4.40 & 5.86 & 6.90 \\
258.095 & 1.69 & $\ldots$ & $5.99(-2)$ & 0.252 & 1.30 & 3.88 & 5.49 \\
290.693 & 1.13 & 1.17 & 0.537 & 0.776 & 0.859 & 0.857 & 0.851 \\
292.801 & 2.40 & $(2.40)$ & 1.18 & 2.37 & 2.68 & 2.65 & 2.63 \\
296.137 & 7.08 & 3.52 & 1.40 & 3.67 & 4.51 & 4.50 & 4.50 \\
341.974 & 1.0 & $\ldots$ & 1.0 & 1.0 & 1.0 & 1.0 & 1.0 \\
344.958 & 0.588 & $\ldots$ & 0.570 & 0.571 & 0.571 & 0.570 & 0.570 \\
345.130 & 2.41 & $\ldots$ & 0.993 & 2.08 & 2.26 & 2.23 & 2.20 \\
349.872 & 4.76 & $\ldots$ & 0.697 & 2.98 & 3.93 & 3.96 & 3.98
\end{tabular}

$\dagger \mathrm{MH}$ solar data from Malinovsky \& Heroux. The MH data are forced to agree with SERTS for the $292.801 \AA$ line.

$\ddagger$ blend

instrumental calibration problems, or inaccurate atomic physics. They may also result from nonequilibrium conditions in the plasma; equilibrium is usually assumed, but not demonstrated. One of the perplexing problems concerns lines of $\mathrm{Fe}$ IX. The Fe IX resonance line at $171.075 \AA$ is one of the strongest lines in the coronal solar spectrum, and it is a strong line in stellar spectra of stars such as Procyon. At longer wavelengths there are two other weaker Fe IX lines at $241.739 \AA$ and $244.912 \AA$ due to intersystem transitions to the ground state. The ratio of these two intersystem lines is an excellent electron density diagnostic for solar and stellar flare densities (e.g., Feldman, Doschek, $\&$ Widing 1978). However, the calculated ratio of the intersystem line intensities to the resonance line intensity is too large by about a factor of 5 to 10 in solar spectra. The reason for this discrepancy is unclear.

$\mathrm{C}$ I isoelectronic sequence ions are relatively simple from the atomic physics standpoint compared to the ions, Fe IX - Fe XVI, which produce strong line emission in the EUV, and which are strong in $E U V E$ spectra of stars such as Procyon. For these ions, better atomic data are needed; however, some data exist and have been used in the analysis of $E U V E$ and solar spectra. There are some excellent density diagnostics for stellar atmospheres. For example, Bhatia \& Doschek (1995) have recently completed calculations for Fe X, adopting a 54 level ion. Fe $\mathrm{X}$ provides the excellent density diagnostic ratio, 175.266 $\AA / 174.534 \AA$. This ratio has been used by Young et al. (1995) to derive solar densities from SERTS spectra, and by Drake, Laming, \& Widing (1995) to derive a density for the atmosphere of Procyon using EUVE spectra.

It is important for the user of atomic data to be aware of the approximations that are adopted in different atomic physics calculations. In the $\mathrm{Fe} \mathrm{X}$ example discussed above, it is important to include resonance contributions in order to obtain an accurate density diagnostic. Resonance excitation of a particular excited level is produced by dielectronic capture onto an ion, followed by autoionization leaving the ion in the particular excited state, or in an excited state that can decay into the level in question. This process can be a very important excitation mechanism in some cases. For Fe X, this is discussed and illustrated by Young et al. (1995). Neglecting resonances in the Fe X line ratio mentioned 
above would lead to an overestimate of density by about a factor of 2.5 over the most sensitive range of the line ratio.

\section{New Solar Flare Results from Yohkoh}

The Yohkoh spacecraft contains high spatial and time resolution soft and hard X-ray telescopes, along with a set of wide band X-ray spectrometers and four highly sensitive Bragg crystal spectrometers. Details of the instrumentation can be found in Solar Physics (vol 136, no. 1, 1991). The combination of high spatial and spectral resolution X-ray instrumentation, coupled with high time resolution and sensitivity, has not occurred previously in solar flare space missions. I will briefly discuss three areas of solar flare research: (1) the dynamics of multimillion degree plasmas, (2) the association of high speed soft X-ray upflows with hard X-ray emission, and, (3) the morphology of soft X-ray flare loops.

In flare numerical simulation models, the soft X-ray flare is formed by the ablation of hot plasma from the chromosphere (chromospheric evaporation). This evaporation might be caused by beams of high energy nonthermal electrons or protons that are accelerated in flaring magnetic flux loops, or by a conduction front due to plasma heating in the loops. In either case, the models predict that X-ray spectral line profiles should be primarily Doppler blueshifted at flare onset, if the flare occurs well within the solar disk (in order to observe the Doppler shift). The Bragg crystal spectrometer (BCS) package on Yohkoh was specifically designed for high sensitivity, in order to obtain statistically meaningful line profiles at flare onset and thereby provide an observational test of the models. (The chromospheric evaporation model is not universally accepted, e.g., see Feldman 1991).

A study by Mariska, Doschek, \& Bentley (1993) of 219 flares observed by BCS has shown that intense blueshifted line components (relative to a stationary spectral component) occur in only about 10 percent of all flares. In the majority of flares, a relatively intense stationary spectral component exists even at flare onset. The presence of this component is difficult to understand in terms of the numerical simulation models. The result does not imply that chromospheric evaporation is not a source of any coronal mass supply, but it does imply that some other physical processes are also at work and that these have not been properly accounted for in the detailed modelling.

In the subset of flares that do show intense blueshifted components, these components first appear at flare onset and disappear or become quite weak at flare maximum and in the decay phase. Therefore, to observe solar-type dynamical activity in stellar spectra, high time resolution over the rise phase of a stellar flare is needed. Ca XIX profiles, and the integrated Ca XIX spectral intensity, are shown in Figure 2 for a solar-type flare exhibiting intense blueshifts at flare onset. The profiles are normalized to the same peak intensity in order to facilitate comparison of line shapes. The histogram spectrum is a typical decay phase spectrum without a blueshifted or significantly Doppler broadened component. These profiles can be easily scaled to EUV wavelengths in order to infer the spectral and time resolution needed to observe upflowing plasma with future EUV experiments. In the beam model of solar flares, the chromospheric heating resulting in evaporated plasma is produced by high energy accelerated particles that heat the chromosphere by Coulomb collisions. Electrons in these beams also produce hard X-ray bursts by thick target Bremsstrahlung. This implies a close correlation between the onset times of hard X-ray bursts and upflowing plasma that emits soft X-rays. These times have been known to be correlated from previous Solar Maximum Mission observations, but with Yohkoh it has been possible to investigate the correlation with much better time resolution. The onset times of the hard X-ray bursts and high speed upflows, i.e., 

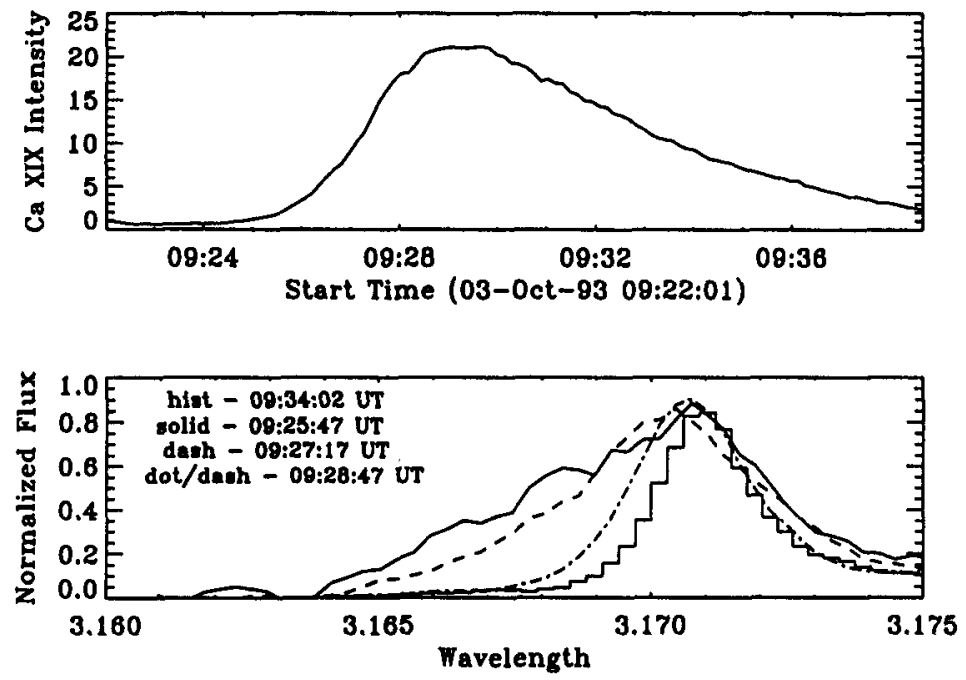

FIGURE 2. Ca XIX resonance line profiles and integrated light curve

plasma with speeds greater than about $400 \mathrm{~km} \mathrm{~s}^{-1}$, are very well-correlated, to within a few seconds, as illustrated in Figure 3 (Bentley et al. 1994). Investigation of Yohkoh soft $\mathrm{X}$-ray images reveals areas with light curves similar to the hard $\mathrm{X}$-ray bursts (Hudson et al. 1994), and these occur at the footpoints of loops. Although some aspects of beam model physics appear verified by these observations, there are some significant problems. In some cases, the footpoint locations do not seem well-connected to the brightest soft $\mathrm{X}$-ray features, making it difficult to understand how evaporation from these footpoints could have filled the soft $\mathrm{X}$-ray loop. An examination of the entire soft X-ray line profile at flare onset (including speeds less than $400 \mathrm{~km} \mathrm{~s}^{-1}$ reveals in some cases blueshifts that can occur up to $100 \mathrm{~s}$ before the soft X-ray flare (Plunkett \& Simnett 1994). This may imply that protons, in addition to electrons, are an important source of energy during the impulsive phase of flares (Plunkett \& Simnett 1994). Perhaps most importantly, a region of apparent confined high energy particles, close to, but outside the soft X-ray emitting loop or loops, has been observed for some flares (Masuda et al. 1994). It is difficult to explain confined high energy particles in the corona, since it always seems possible for the particles to expand in some direction or directions along magnetic field lines.

There is a similar confinement problem with the soft X-ray flare. One of the most perplexing results from Yohkoh concerns the morphology of soft X-ray flare loops. Because a flare loop is essentially a magnetic tube with the field lines oriented in the direction of the major loop axis, models of gas behavior in loops predict a smooth distribution of plasma, i.e., constant pressure along the loop axis except for short time periods in which nonequilibrium dynamical effects may exist. However, observations by the Yohkoh soft X-ray telescope (SXT) of many fiare loops (see Figure 4) show that a confined bright region is often found at the tops of flare loops (Acton et al. 1992; Feldman et al. 1994; Doschek, Strong, \& Tsuneta 1995), and these regions exist from near flare onset to late in the flare decay phase. Measurements of temperature and emission measure along the flare loop axis indicate that these bright regions represent a region of enhanced pressure 


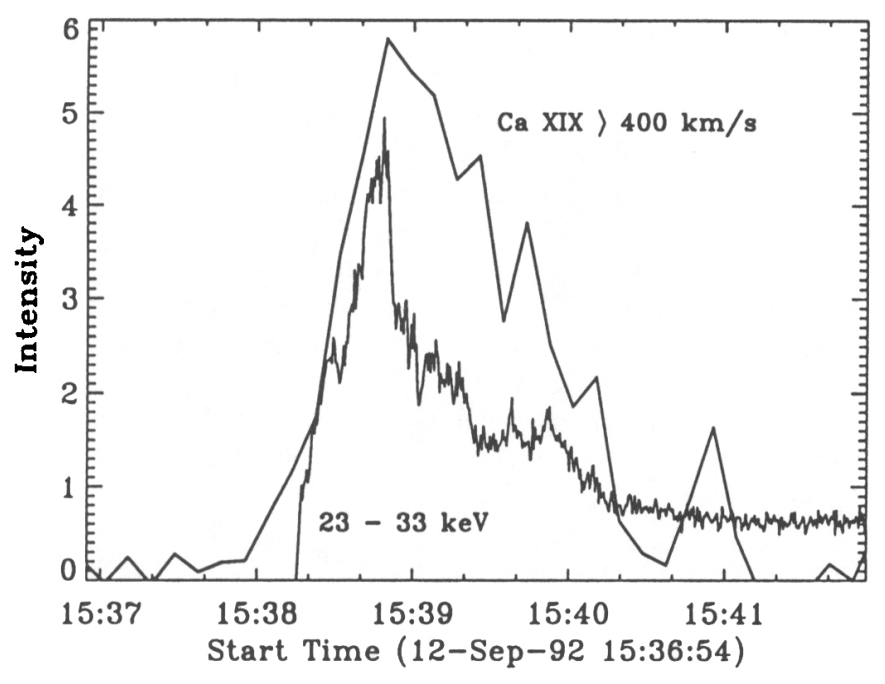

Figure 3. Ca XIX and hard X-ray light curves. The Ca XIX curve represents plasma moving at Doppler speeds greater than $400 \mathrm{~km} \mathrm{~s}^{-1}$

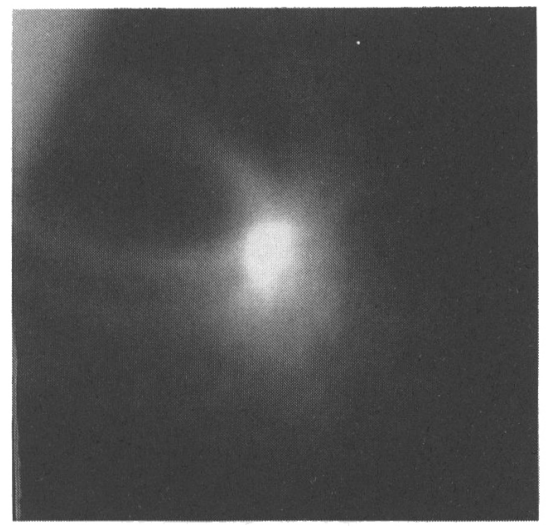

FIGURE 4. SXT LDE image (2 Nov. 1992, 10:13:36 UT) 
in the loop. It is difficult to understand how such a region can exist for long time periods, and explaining these structures is now a current and important problem in flare modelling.

Some indication of these bright regions was noticed in previous Skylab observations of long duration events (LDEs) (Kahler 1977; Vorpahl, Tandberg-Hanssen, \& Smith 1977), which are very large flares, spatially, and last for many hours. They were detected because of the size and longevity of LDEs, and because the bright regions are particularly noticeable in these events. The dramatic illustration of a bright knot shown in Figure 4 is an SXT image of an LDE flare. However, the Yohkoh observations show that these bright regions are common properties of compact flares as well.

In summary, the observations from Yohkoh support some features of solar models, in the sense that evidence for high energy particle beams and evaporating plasma is seen in flares. But the details of the images and spectra conflict strongly in many cases with the details of the models, and the apparent longevity of confined bright regions, observed in both soft and hard X-ray images, appears to require significant revision or additions in the physics of flare models.

The author was supported by a NASA Grant from the Astrophysics Data Program. The author thanks J. Mariska for assistance with manuscript preparation.

\section{REFERENCES}

Acton, I. W., Bruner, M. E., Brown, W. A., Fawcett, B. C., Schweizer, W., \& Speer, R. J. 1985, ApJ, 291, 865

Acton, L. W. ET AL. 1992, Publ. Astron. Soc. Japan, 44, L71

Bentley, R. D., DoscheK, G. A., Simnett, G. M., Rilee, M. L., Mariska, J. T., Culhane, J. L., Kosugi, T., \& Watanabe, T. 1994, ApJL, 421, L55

Bhatia, A. K. \& Doschek, G. A. 1993, Atomic Data and Nuclear Data Tables, 55, 281

Bhatia, A. K. \& DoscheK, G. A. 1995, Atomic Data and Nuclear Data Tables, in press

DoscheK, G. A., Strong, K. T., \& Tsuneta, S. 1995, ApJ, 440, 370

Drake, J. J., LAming, J. M., \& Widing 1995, ApJ, 443, 393

Feldman, U. 1991, Flare Physics in Solar Activity Maximum 22, ed. Y. Uchida, R.C. Canfield,

T. Watanabe, \& E. Hiei, Springer, Lecture Notes in Physics, 387, 146

Feldman, U., Doschek, G. A., \& Wming, K. G. 1978, ApJ, 219, 304

Feldman, U., Seely, J. F., Doschek, G. A., Strong, K. T., Acton, L. W., Uchida, Y., \& TSUneta, S. 1994, ApJ, 424, 444

Hudson, H. S., Strong, K. T., Dennis, B. R., Zarro, D., Inda, M., Kosugi, T., \& Sakao, T. 1994, ApJL, 422, L25

KAHLER, S. 1977, ApJ, 214, 891

Malinovsky, M. \& Heroux, L. 1973, ApJ, 181, 1009

Mariska, J. T., Doschek, G. A., \& Bentley, R. D. 1993, ApJ, 419, 418

Masuda, S., Kosugi, T., Hara, H., Tsuneta, S., \& Ogawara, Y. 1994, Nature, 371, 495 Plunkett, S. P. \& Simnett, G. M. 1994, Solar Phys., 155, 351

Thomas, R. J. \& NeUperT, W. M. 1994, ApJS, 91, 461

Vorpahl, J. A., Tandberg-Hanssen, E., \& SMith, J. B., Jr. 1977, ApJ, 212, 550

Young, P. R., Mason, H. E., Bhatia, A. K., Doschek, G. A., \& Thomas, R. J. 1995, these proceedings 